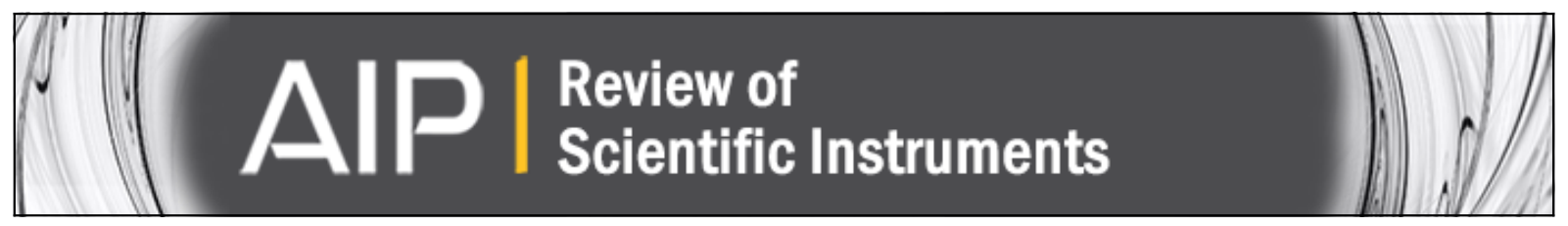

Note: Significant increase to the temporal resolution of 2D X-ray detectors using a novel beam chopper system

Stefan Küchemann, Carsten Mahn, and Konrad Samwer

Citation: Review of Scientific Instruments 85, 016105 (2014); doi: 10.1063/1.4863656

View online: http://dx.doi.org/10.1063/1.4863656

View Table of Contents: http://scitation.aip.org/content/aip/journal/rsi/85/1?ver=pdfcov

Published by the AIP Publishing

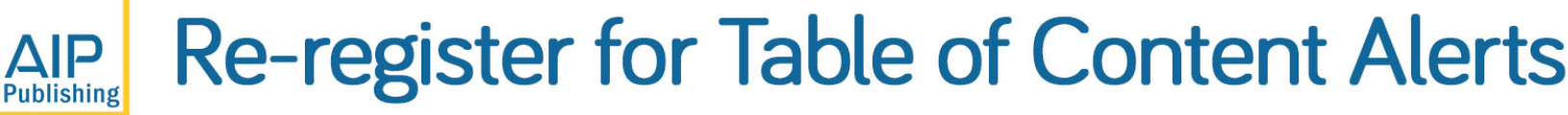




\title{
Note: Significant increase to the temporal resolution of 2D X-ray detectors using a novel beam chopper system
}

\author{
Stefan Küchemann, Carsten Mahn, and Konrad Samwer \\ 1. Physikalisches Institut, Georg-August-Universität Göttingen, Friedrich-Hund-Platz 1, \\ 37077 Göttingen, Germany
}

(Received 12 November 2013; accepted 16 January 2014; published online 31 January 2014)

\begin{abstract}
The investigation of short time dynamics using X-ray scattering techniques is commonly limited either by the read out frequency of the detector or by a low intensity. In this paper, we present a chopper system, which can increase the temporal resolution of 2D X-ray detectors by a factor of 13. This technique only applies to amorphous or polycrystalline samples due to their circular diffraction patterns. Using the chopper, we successfully increased the temporal resolution up to $5.1 \mathrm{~ms}$ during synchrotron experiments. For the construction, we provide a mathematical formalism, which, in principle, allows an even higher increase of the temporal resolution. (C) 2014 AIP Publishing LLC. [http://dx.doi.org/10.1063/1.4863656]
\end{abstract}

Structural studies of metallic glasses have gained a wide interest. ${ }^{1,2}$ In binary and ternary systems, these studies are limited to temperatures close to the glass transition temperature due to the rapid incipient crystallization in the supercooled liquid region. However, simulations and measurements on five-component glass formers show interesting features, like strong-fragile transitions and liquid-liquid phase transitions in this hardly accessible temperature regime. ${ }^{3,4}$ Up to now, there are CCD flat panel X-ray detectors, such as the Rayonix MX-HS series, ${ }^{5}$ with a frame rate of $140 \mathrm{~Hz}$ and pixel detectors with a frame rate of $500 \mathrm{~Hz}$ from Pilatus ${ }^{6}$ and a frame rate of $4.5 \mathrm{MHz}$ from the European XFEL. ${ }^{7}$ Generally, these detectors are costly and limited to energies below $25 \mathrm{keV}$. In a recent experiment which studied fast dynamics of metallic glasses, it was necessary to increase the temporal resolution of the 2D flat panel detector from Perkin Elmer XRD 1621 of $66 \mathrm{~ms}(15 \mathrm{~Hz})$ available at beamline P07 at PETRA III, DESY. The X-ray beam was set to an energy of $70 \mathrm{keV}$ which corresponds to a wavelength of $0.17712 \AA$. Due to the isotropic, disordered structure of these materials, the X-ray diffraction patterns have a circular shape and contain nearly the same information in each direction from the beam center. Thus, in order to obtain momentary structural information, it is only necessary to know the diffraction in one direction from the beam center and it is not necessary to integrate over the entire diffraction ring. Therefore, we installed the chopper system between the sample and the detector so that the diffracted beam is temporally partly blocked by the chopper. The chopper divides the diffraction pattern into 13 sections and these sections are illuminated successively. Hence, with the aid of the chopper system, the temporal resolution could be increased from $66 \mathrm{~ms}(15 \mathrm{~Hz})$ up to $5.1 \mathrm{~ms}(195 \mathrm{~Hz})$. The whole system consists of two axes (labeled with "Axis A" and "Axis B") and the chopper suspension (Fig. 1(a)). There are two chopper discs integrated in the chopper suspension, which rotate in opposite directions at different frequencies. Axis A is driven by the brushless EC servomotor nanotech DB87M01-S with a nominal torque of $1.4 \mathrm{Nm}$. It rotates with the same frequency as the fast chopper disc. On this axis, there are two gears and two discs for photo sensors attached. The sensors are used to record the moment when a new section on the detector starts to be illuminated. The first gear on the axis has 40 teeth (and a diameter of $\mathrm{d}=40 \mathrm{~mm}$ ) and the second one has $72(\mathrm{~d}=110 \mathrm{~mm})$. Both are connected to gears on axis $\mathrm{B}$, the first is directly connected to another gear with 124 teeth $(\mathrm{d}=130 \mathrm{~mm})$ and the second one via a gear belt to a gear with 18 teeth $(\mathrm{d}=25 \mathrm{~mm})$. The latter gear is attached to the axis via a ball bearing, so that it can rotate in the opposite direction than this axis itself. Both gears on the second axis are coupled via gear belts to the outer suspension of the chopper. The choppers are mounted on the outer edge to a gear with 72 teeth instead of a central suspension in order not to lose any information close to the beam center (see Fig. 1(b)) and to achieve a higher mechanical stability. The chopper discs are made of lead and rotate in opposite directions. The discs have a thickness of $3 \mathrm{~mm}$. At an energy of the X-ray beam of $70 \mathrm{keV}$, this thickness causes a decrease of the intensity by a factor of $3.18 \times 10^{-6} .8$ Apart from this, the discs have an outer diameter of $100 \mathrm{~mm}$ and a remaining lead center with a diameter of $3 \mathrm{~mm}$. The first, fast rotating disc has one slit and the second slow one has six slits (Fig. 1(d)). These numbers are variable but this combination has been proven to fulfill the required conditions discussed below while maintaining a sufficient mechanical stability of the lead choppers. The frequency of the fast chopper is adjusted to be two times the read out frequency of the detector. Figure 1(c) shows a photograph of the chopper system used at the beamline P07 at PETRA III, DESY. For the construction, it is helpful to know that the ball bearings on the first and on the second axis must be made for $1800 \mathrm{rpm}$. The ball bearing, which is used to attach the first gear on axis B, needs to stand $3600 \mathrm{rpm}$ since the gear rotates in the opposite direction than its axis. Additionally, in order to keep the gear belts under tension, we attached the chopper suspension to a movable rail and added another movable clamping fixture for the gear belt between axis A and axis B. Please note that this clamping fixture is not included in the schematic drawings in Figs. 1(a) and 1(b), but in the photograph in Fig. 1(c). On top of the first, fast rotating chopper 
a)
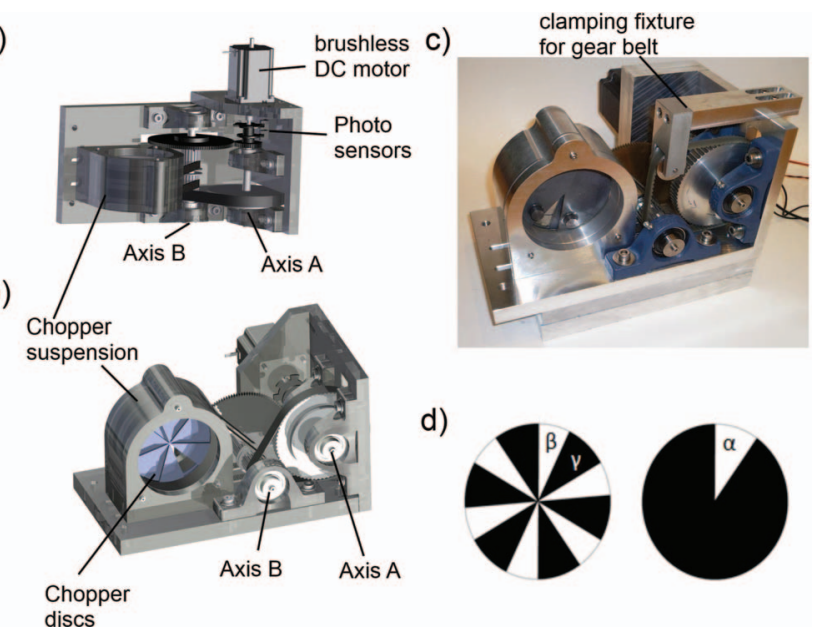

d)

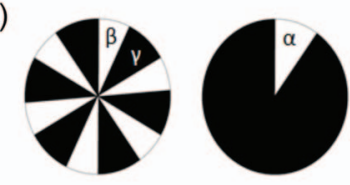

FIG. 1. (a) Technical drawing of the two choppers rotating in opposite directions (top view). (b) Technical drawing of the two choppers (front view). (c) Photograph of the assembled chopper system. (d) Schematic drawing of the two chopper discs. The second, slow chopper has 6 slits with an opening angle of $\beta=25^{\circ}$, accordingly $\gamma=35^{\circ}$. The first, fast chopper has only one slit with an opening angle of $\alpha=35^{\circ}$ fulfilling Eq. (2).

disc with only one slit, we also added two round lead pieces in order to compensate the asymmetric mass distribution due to the single slit of this chopper disc. In order to check the performance of the chopper, we calculated the position of the slits as function of time (Fig. 2). The grey regions correspond to the positions of the slits at a certain time. White regions occur at the angle and time where two slits overlap and thus this particular area on the detector is illuminated. An overlap of the angles of the white regions would lead to the situation where a certain angle on the detector contains information from different time zones. This means that a discrimination between the information during the analysis would not be

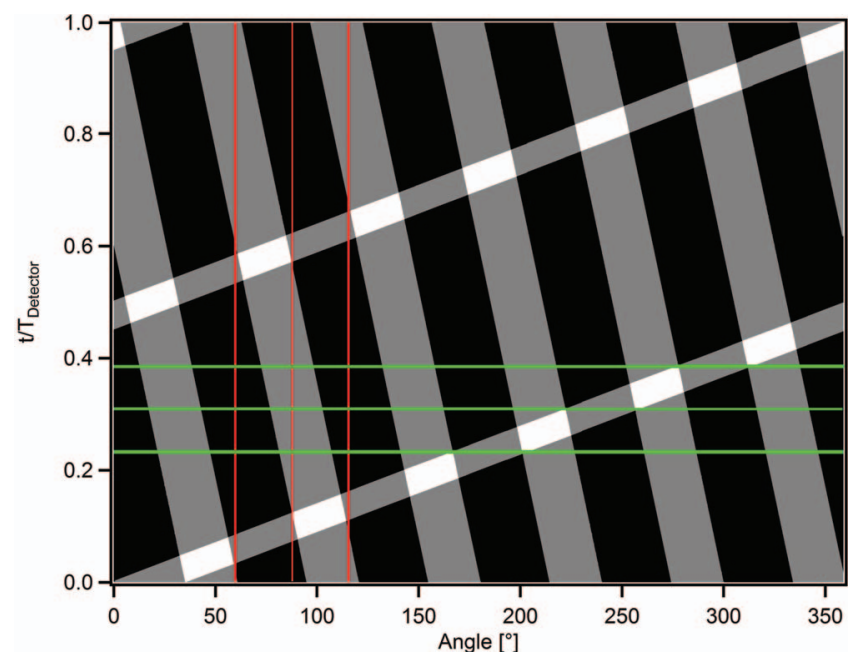

FIG. 2. The progress of the slits of the two choppers is plotted in grey. The white areas, which correspond to the time and angle where two slits, one from each chopper, overlap, do not show an overlap in time (indicated by horizontal, green lines) or in the angle (indicated by vertical, red lines). This means that two illuminated sections are clearly separated in space and do not contain the same information of the temporal evolution. Moreover, all information of the temporal evolution is captured since there is no gap between two subsequent white areas in time.

possible. Therefore, it is essential that the angles of the white sections do not overlap (as it is shown by the red lines in Fig. 2). This condition is manifested in the following inequation, which relates the fraction between the rotational periods of the two choppers $\frac{T_{2}}{T_{1}}$ and the sizes of the slits,

$$
\frac{360^{\circ}-\alpha}{\beta} \geq \frac{T_{2}}{T_{1}} \geq \frac{360^{\circ}+\alpha}{\gamma}
$$

where $\alpha$ is opening angle of the only slit of the fast chopper, $\beta$ is the opening angle of the six slits of the slow chopper, and $\gamma=360^{\circ} / \mathrm{n}-\beta$ reflects the angle between two slits of the slow chopper. The factor $n$ is the number of slits in the slow chopper 2. Additionally, we tried to avoid that the times of the white sections of different regions overlap (as indicated by the green lines in Fig. 2). This is not an essential condition but simplifies the analysis. The condition relating the opening angles of the slits of the two choppers is reflected
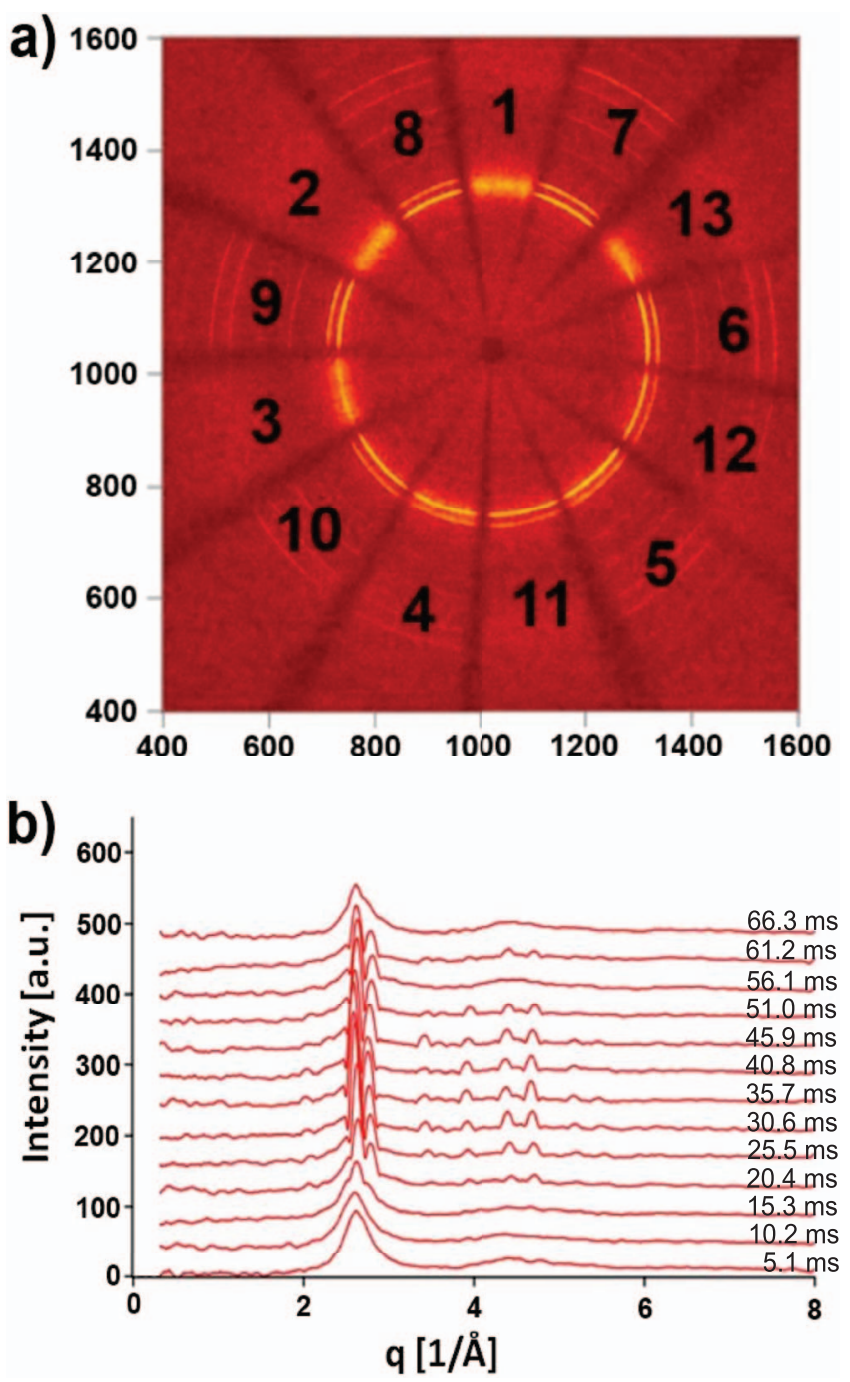

FIG. 3. The 2D detector image shows a clear separation between the different sections (a). The numbers indicate the temporal order. The integrated intensities are plotted in panel (b) in the correct temporal order. Note that section 13 also contains information about the beginning of the temporal evolution within one detector image, since the start of the first section does not match exactly with the start of the illumination of detector image. 
in the equation:

$$
\beta=\frac{360^{\circ}}{n}-\alpha
$$

When constructing the chopper system, one can choose the parameters in the way that a section starts exactly at the same angle and time where the previous one ended. So, there is not gap neither in time nor in the angle between two adjacent white regions in Fig. 2. This choice is considered to be the most efficient case since the sections on the detector are largest and longest illuminated. It occurs when the two expressions on the left and on the right side in Eq. (1) are equal and can be solved when considering Eq. (2). This combination results in $\alpha=32.7273$ and $\beta=27.2727$. These values, in turn, lead to the ideal fraction between the rotational periods of $\frac{T_{2}}{T_{1}}=12$. This efficient construction has a technical disadvantage, which is the difficulty to discriminate between two neighboring sections. Therefore, we slightly varied the parameters to the values of $\alpha=35^{\circ}$ and $\beta=25^{\circ}$ and a fraction between the rotational periods of $\frac{T_{2}}{T_{1}}=12.4$. Thus, there is always a little gap remaining between two sections in the detector image but there is no gap in time between two sections (see Fig. 3). The chopper system was first used during our beamtime at P07 at DESY in Hamburg in 2012. We investigated the crystallization of metallic glasses during fast heating. At high heating rates the crystallization process takes place rapidly. With the aid of the chopper, we were able to resolve the formation and growth of crystals even at high heating rates (Fig. 3). The numbers in Fig. 3(a) show that neighboring sections are not temporally sequenced but the second-next neighboring ones are. The chopper system enables a significant increase of the temporal resolution, in our case, by a factor of 13. Particularly for high-energy synchrotron beams, this technique is a simple and cost-efficient way to increase the temporal resolution. The signal to noise ratio is considerably decreased, in the current setup by a factor of 169 . However, the signal to noise ratio is sufficient to clearly resolve the crystallization behavior of metallic glasses. In principle, the chopper system can be applied to any, also faster framing 2D detector. Given a different detector, the speed of the motor must be set to be as twice as high as the read out frequency. Currently, the installed motor, with $3000 \mathrm{rpm}$, limits the use of the chopper system to detectors with a temporal resolution of $40 \mathrm{~ms}(25$ $\mathrm{Hz}$ ). However, there are much faster accurate brushless motors available. For example, with a motor of $13000 \mathrm{rpm}$ and appropriate ball bearings the chopper system could be used for detectors with a resolution of about $9.2 \mathrm{~ms}(108 \mathrm{~Hz})$ and accordingly increase this temporal resolution up to $0.7 \mathrm{~ms}$ $(1408 \mathrm{~Hz})$. This value even exceeds the highest temporal resolution of current Pilatus detectors. ${ }^{6}$ Given a beam with a higher intensity, the temporal resolution could also be increased by modifying the number of slits $n$ of the second slow chopper. The fraction between the rotational periods and the angle of the slits can be calculated using Eqs. (1) and (2).

The authors would like to acknowledge the remarkable effort of the central workshop of the Physics Department at the University of Göttingen regarding the setup of the chopper and also thank the beamline team of P07 at Petra III for their kind and helpful assistance during the beamtime.

${ }^{1}$ Y. Q. Cheng, E. Ma, and H. W. Sheng, Phys. Rev. Lett. 102, 245501 (2009).

${ }^{2}$ G. Duan, D. Xu, Q. Zhang, G. Zhang, T. Cagin, W. L. Johnson, and W. A. Goddard, Phys. Rev. B 71, 224208 (2005)

${ }^{3}$ S. Wei, F. Yang, J. Bednarcik, I. Kaban, O. Shuleshova, A. Meyer, and R. Busch, Nat. Commun. 4, 2083 (2013)

${ }^{4}$ K. N. Lad, N. Jakse, and A. Pasturel, J. Chem. Phys. 136, 104509 (2012).

${ }^{5}$ See rayonix webpage: http://rayonix.com/products/mx-hs-series/,11/1/ 2014 for the technical specification of the detectors in the Rayonix MXHS series.

${ }^{6}$ See dectris webpage of Pilatus3 detectors: https://www.dectris.com/ pilatus3_features.html\#main_head_navigation, 10/23/2013 for the specification of the Pilatus 3 detector series.

${ }^{7}$ J. Becker, D. Greiffenberg, U. Trunk, X. Shi, R. Dinapoli, A. Mozzanica, B. Henrich, B. Schmitt, and H. Graafsma, Nucl. Instrum. Methods Phys. Res. A 694, 82-90 (2012).

${ }^{8}$ See NIST webpage: http://physics.nist.gov/PhysRefData/XrayMassCoef/ ElemTab/z82.html, 5/6/2013 for the mass attenuation coefficient of lead at an energy of $70 \mathrm{keV}$, which is $\mu / \rho=3.72 \mathrm{~g} / \mathrm{cm}^{2}$. 